\title{
The Knowledge Tightness of Parallel Zero-Knowledge
}

\author{
Kai-Min Chung ${ }^{\star}$, Rafael Pass ${ }^{\star \star}$, and Wei-Lung Dustin Tseng \\ Department of Computer Science, Cornell University, Ithaca, NY, USA \\ \{chung, rafael, wdtseng\}@cs. cornell.edu
}

\begin{abstract}
We investigate the concrete security of black-box zeroknowledge protocols when composed in parallel. As our main result, we give essentially tight upper and lower bounds (up to logarithmic factors in the security parameter) on the following measure of security (closely related to knowledge tightness): the number of queries made by black-box simulators when zero-knowledge protocols are composed in parallel. As a function of the number of parallel sessions, $k$, and the round complexity of the protocol, $m$, the bound is roughly $k^{1 / m}$.

We also construct a modular procedure to amplify simulator-query lower bounds (as above), to generic lower bounds in the black-box concurrent zero-knowledge setting. As a demonstration of our techniques, we give a self-contained proof of the $o(\log n / \log \log n)$ lower bound for the round complexity of black-box concurrent zero-knowledge protocols, first shown by Canetti, Kilian, Petrank and Rosen (STOC 2002). Additionally, we give a new lower bound regarding constant-round black-box concurrent zero-knowledge protocols: the running time of the black-box simulator must be at least $n^{\Omega(\log n)}$.
\end{abstract}

Keywords: Zero-Knowledge, Knowledge Tightness, Concrete Security, Concurrent Zero-Knowledge Lower Bounds.

\section{Introduction}

Zero-knowledge interactive proofs, introduced by Goldwasser, Micali and Rackoff GMR89 are paradoxical constructions allowing one player (called the prover) to convince another player (called the verifier) of the validity of a mathematical statement $x \in L$, while providing no additional knowledge to the verifier. In addition to being an independent construction of interest, zero-knowledge have become an extremely useful tool in construction of numerous cryptographic protocols.

^ Chung is supperted by a Simons Foundation Fellowship.

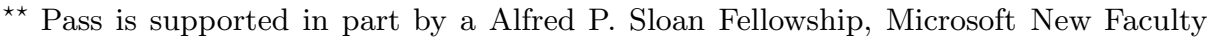
Fellowship, NSF CAREER Award CCF-0746990, AFOSR YIP Award FA9550-101-0093, and DARPA and AFRL under contract FA8750-11-2-0211. The views and conclusions contained in this document are those of the authors and should not be interpreted as representing the official policies, either expressed or implied, of the Defense Advanced Research Projects Agency or the US government. 
A fundamental question regarding zero-knowledge protocols is whether their composition remains zero-knowledge. In theoretical constructions as well as in practice, a zero-knowledge protocol is sometimes composed in parallel (to amplify soundness or to improve efficiency, for example). It is well-known that the definition of zero-knowledge (ZK) is not closed under parallel composition [GK96b. Nevertheless, we know numerous constructions of constant-round zero-knowledge protocols that are secure when composed in parallel [FS90, GK96a, Gol02. As a result, the subject of ZK with respect to parallel composition is widely considered closed.

We turn our attention to another fundamental question regarding zeroknowledge: its knowledge tightness. In its original definition, the zero-knowledge property is formalized by requiring that the view of any probabilistic polynomial time (PPT) verifier $V$ in an interaction with a prover can be "indistinguishably reconstructed" by a PPT simulator $S$ that interacts with no one. Since whatever $V$ "sees" in the interaction can be reconstructed by the simulator, the interaction does not yield any knowledge to $V$ that $V$ can already compute by itself. Because the simulator is allowed to be an arbitrary PPT machine, this traditional notion of ZK only guarantees that the class of PPT verifiers learn nothing.

To more concretely measure the knowledge gained by a particular verifier, Goldreich, Micali and Wigderson GMW91] (see also Gol01]) put forward the notion of knowledge tightness: informally, the "tightness" of a simulation is the ratio of the (expected) running-time of the simulator, divided by the (worst-case) running-time of the verifier. Thus, in a knowledge-tight ZK proof, the verifier is expected to gain no more knowledge than what it could have computed in time closely related to its worst-case running-time. In addition to theoretical interests, the knowledge tightness of a zero-knowledge protocol is a helpful aid for setting the security parameter in practice. It is easy to check that the original zero-knowledge protocols GMR89, GMW91, Blu86] all enjoy constant knowledge tightness. The aforementioned protocols secure under parallel composition FS90, GK96a, Gol02 also enjoy constant knowledge tightness when executed in isolation; however, when composed in parallel, the tightness of these protocols seem increase/loosen linearly (sometimes even quadratically) with respect to the number of parallel sessions (based on the currently known analysis of their simulators)!

Since we do want to execute zero-knowledge protocols in parallel (for instance in the application of secure multi-party computation), a natural question is to ask: how does the knowledge tightness of a protocol vary when we increase the number of parallel repetitions?

\subsection{Our Results}

In this work we give essentially tight upper and lower bounds to the above question. Our results focus on black-box zero-knowledge and "simulator queries", which we explain below.

Informally, a protocol is black-box zero-knowledge if there exists a universal simulator $S$, called the black-box simulator, such that $S$ generates the view of 
any adversarial verifier $V^{*}$ if $S$ is given black-box access to $V^{*}$. Essentially all known constructions of zero-knowledge (with the notable exception of [Bar01]) and all practical zero-knowledge protocols are black-box zero-knowledge. Given a black-box simulator $S$, we focus on bounding the number of black-box queries made by $S$ to a given adversarial verifier $V^{*}$; we refer to this as the simulatorquery complexity. It is easy to see that the number of queries made by a blackbox simulator is closely related to knowledge tightness; in fact, for the case of constant round protocols, they are asymptotically equivalent.

We state our main theorems below:

Theorem 1. Let $n$ be the security parameter. For any $m=m(n)$, there exists a $2 m+7$-round black-box zero-knowledge argument $\Pi$ for all of NP based on oneway functions, with perfect completeness and negligible soundness error, such that for any polynomially bounded $k=k(n)$, the parallel composition of $k$-copies of the protocol, $\Pi^{k}$, remains black-box zero-knowledge with simulator-query complexity $O\left(m k^{1 / m} \log ^{2} n\right)$.

The above theorem can be extended to proofs assuming the existence of collisionresistant hash-functions. We complement Theorem 1 with a lower bound:

Theorem 2. Let $n$ be the security parameter, $L$ be a language, and $m=m(n) \in$ $O\left(\frac{\log n}{\log \log n}\right)$. Suppose $\Pi$ is a $m(n)$-round black-box zero-knowledge argument for $L$ with perfect completeness and negligible soundness error, and suppose there exist a polynomially bounded $k(n) \geq n$ such that the parallel composition of $k$-copies of the protocol, $\Pi^{k}$, remains black-box zero-knowledge with simulatorquery complexity $O\left(k^{1 / m} /\left(\log ^{2} n\right)\right)$. Then, $L \in$ BPP.

For protocols with sub-logarithmic number of rounds, Theorem 1 and 2 are tight up to logarithmic factors in the security parameter; essentially, the simulatorquery complexity is asymptotically close to $k^{1 / m}$ (in most cases, think of $k$ as a low polynomial in $n$ ). We mention that one can achieve simulator-query complexity $O(m)$ (independent of $k$ ) when $m=\omega(\log n)$.

Briefly, our results show that the concrete security of constant-round blackbox zero-knowledge protocols actually decays polynomially in the number of parallel sessions. Fortunately, this decay can be significantly slowed if we consider protocols with more rounds (even if we simply use a large constant $m$ ).

\subsection{Related Works}

While we are unaware of any past work that explicitly studies the knowledge tightness of parallelized zero-knowledge protocols, there are numerous related publications that focus on the composition of zero-knowledge protocols, or on the concrete security of zero-knowledge simulator. Dwork, Naor and Sahai DNS04] introduces the notion of concurrent zero-knowledge protocols; these protocols must stay zero-knowledge even when composed arbitrarily (a strengthening over parallel composition). Micali and Pass [MP06] introduces the notion of precision; in a precise zero-knowledge protocol, the running time of the simulator should 
be closely related to the running time of the adversarial verifier, on a view by view basis 1 (a strengthening over knowledge tightness).

Even with these stronger requirements, Pandey et. al. $\mathrm{PPS}^{+} 08$ is able to construct protocols that are simultaneously precise and (black-box) concurrent zeroknowledge. Note that our results are incomparable with the result of $\mathrm{PPS}^{+} 08$ for many reasons, one of which being that black-box concurrent zero-knowledge protocols require logarithmically many rounds [CKPR01, while our setting is mainly interesting for sub-logarithmic-round protocols. Interestingly, $\mathrm{PPS}^{+} 08$ actually gives a construction of a family of precise concurrent zero-knowledge protocols, with trade-offs between round-complexity and precision, much like our observed trade-off between round-complexity and knowledge tightness for the case of parallelized zero-knowledge.

\subsection{Connection to Concurrent Zero-Knowledge}

We also present a connection from simulator-query lower bounds for zeroknowledge, to round-complexity lower bounds for concurrent zero-knowledge (cZK). Due to lack of space we postpone the result on concurrent zero-knowledge to the full version. We briefly discuss the ideas as follows.

We start by describing the common framework for all known black-box zeroknowledge lower bounds (e.g., [KPR98, Ros00, CKPR01, BL02, Kat08, HRS09]). Let $\Pi$ be a protocol for a language $L$. To show that $\Pi$ cannot be zero-knowledge unless the language $L$ is trivial (i.e., $L \in \mathrm{BPP}$ ), we start by constructing a decision procedure for $L$. Let $S$ be the black-box zero-knowledge simulator of $\Pi$, and let $V^{*}$ be some "hard to simulate" adversarial verifier, and consider the following decision procedure $\mathcal{D}$ : on input $x, \mathcal{D}(x)$ accepts if and only if $S^{V^{*}}(x)$ generates an accepting view of $V^{*}(x)$. Usually, the completeness of $\mathcal{D}$ follows easily from the zero-knowledge property; to show that $\mathcal{D}$ is sound often requires more work. Our query-complexity lower bounds (Theorem2) also follow the same framework. That is, we construct some adversarial verifier $V_{\text {para }}^{*}$ that schedules multiple sessions in parallel, and show that for any zero-knowledge simulator $S$ with appropriately bounded query-complexity, if $x \notin L$, then $S^{V_{\text {para }}^{*}}(x)$ cannot generate an accepting view of $V_{\text {para }}^{*}(x)$.

Inspired by the work of Canetti, Kilian, Petrank and Rosen CKPR01, we next present a modular construction of a concurrent adversarial verifier $V_{\text {conc }}^{*}$ whose purpose is to amplify query-complexity lower bounds of more basic verifiers. For example, consider $V_{\text {para }}^{*}$, an adversarial verifier that is restricted to parallel composition. Our modular construction would take $V_{\text {para }}^{*}$ as input, and output an adversarial verifier $V_{\text {conc }}^{*}=V_{\text {conc }}^{*}\left(V_{\text {para }}^{*}\right)$ that, among other things, nests multiple incarnations of $V_{\text {para }}^{*}$ in a way that takes full advantage of the concurrent scheduling. Under appropriate parameters, our analysis would conclude that for any zeroknowledge simulator $S$ with polynomially bounded query-complexity, if $x \notin L$,

${ }^{1}$ For example, to achieve precision 2, if the simulator $S$ generates a view of $V^{*}$ and the running time of $V^{*}$ on that view is $T$, then the simulator $S$ must have run in time $2 T$. 
then $S_{\text {conc }}^{V^{*}}(x)$ cannot generate an accepting view of $V_{\text {conc }}^{*}(x)$ (recall again that this is the key step for most zero-knowledge lower bounds).

To demonstrate our framework, we re-prove the result of [CKPR01 - a $o(\log n / \log \log n)$ round-complexity lower bound for black-box concurrent zeroknowledge (the currently best known round-complexity lower bound); we believe the resulting analysis is quite clean. We also give a second lower bound concerning constant-round cZK protocols:

Theorem (Informal). Let $L$ be a non-trivial language, and let $\Pi$ be a constantround black-box concurrent zero-knowledge protocol with a potentially possibly super-polynomial time simulator. Then the simulator must run in time $n^{\Omega(\log n)}$.

Incidentally, Pass and Venkitasubramaniam PV08] do construct constant-round black-box concurrent zero-knowledge protocols for all of NP in the model where both the simulator and the adversarial verifier runs in quasi-polynomial time $n^{\text {poly }(\log n)}$.

We also find our modular framework satisfying on a philosophical level: it serves as an framework in which lower bounds for restricted compositions of zero-knowledge (in this example parallel composition) can be transformed into lower bounds for zero-knowledge in the fully concurrent setting. A similar and celebrated example occurs in the work of Goldreich Gol02, where it is shown that constructions of zero-knowledge protocols secure under parallel composition directly leads to constructions of concurrent zero-knowledge protocols secure in the timing model.

\section{Preliminaries}

We use $\mathbb{N}$ to denote the natural numbers $\{0,1, \ldots\},[n]$ to denote the set $\{1, \ldots, n\}$, and $|x|$ to denote the length of a string $x \in\{0,1\}^{*}$. By $\mathrm{ngl}(\mathrm{n})$, we mean a function negligible in $n$ (i.e., $\left.1 / n^{\omega(1)}\right)$. We assume familiarity with indistinguishability.

Interactive Protocols. An interactive protocol $\Pi$ is a pair of interactive Turing machines, $(P, V)$, where $V$ is probabilistic polynomial time (PPT). $P$ is called the prover, while $V$ is called the verifier. $\langle P, V\rangle(x)$ denotes the random variable (over the randomness of $P$ and $V$ ) representing $V$ 's output at the end of the interaction on common input $x$. If additionally $V$ receives auxiliary input $z$, we write $\langle P(x), V(x, z)\rangle$ to denote $V$ 's output. We assume WLOG that $\Pi$ starts with a verifier message and ends with a prover message, and say $\Pi$ has $k$ rounds if the prover and verifier each sends $k$ messages alternately. A full or partial transcript of $\Pi$ is a sequence of alternating verifier and prover messages, $\left(v_{1}, p_{1}, \ldots\right)$, where $v$ denotes verifier messages and $p$ denotes prover messages.

We may compose an interactive proof in parallel. Let $\Pi^{k}=\left(P^{k}, V^{k}\right)$ be the parallel composition of $k$ copies of $\Pi$; that is, each prover and verifier message in $\Pi^{k}$ is just concatenation of $k$ independent copies of the corresponding message in $\Pi$. Upon completion, $V^{k}$ accepts if and only if all $k$ sessions are accepted by $V$. We note that an adversarial verifier may choose to abort in one session but not another. 
Zero Knowledge Protocols. In the setting of zero knowledge, we consider an adversarial verifier that attempts to "gain knowledge" by interacting with an honest prover. An adversarial verifier $V^{*}$ is a probabilistic polynomial time machine that, on common input $x$ and auxiliary input $z$, interacts with the prover $P$. Let $\operatorname{View}_{V^{*}}^{P}(x, z)$ be the random variable that denotes the view of $V^{*}$ in an interaction with $P$ (this includes the random coins of $V^{*}$ and the messages received by $V^{*}$ ).

A black-box simulator $S$ is a probabilistic polynomial time machine that is given black-box access to $V^{*}$ (written as $S^{V^{*}}$ ). Formally, $S$ fixes the random coins $r$ of $V^{*}$ a priori, and $S$ is allowed to specify a valid partial transcript $\tau=\left(v_{1}, p_{1}, \ldots, p_{i}\right)$ of $V_{r}^{*}$, and query $V_{r}^{*}$ for the next verifier message $v_{i+1}$. Here, $\tau$ is valid if it is consistent with $V_{r}^{*}$, i.e., each verifier message $v_{j}$ in $\tau$ is what $V_{r}^{*}$ would have responded given the previous prover messages $p_{1}, \ldots, p_{j-1}$ and the fixed random tape $r$. Note that $S$ is allowed to "rewind" $V^{*}$ by querying $V^{*}$ with different partial transcripts that shares a common prefix.

Intuitively, an interactive proof is zero-knowledge $(Z K)$ if the view of any adversarial verifier $V^{*}$ can be generated by a simulator. The formal definition follows.

Definition 3 (Black-Box Zero-Knowledge [GMR89, GO94]). Let $\Pi=$ $\langle P, V\rangle$ be an interactive proof (or argument) for a language $L$. $\Pi$ is blackbox zero-knowledge if there exists a black-box simulator $S$ such that for every common input $x$, auxiliary input $z$ and every adversary $V^{*}, S^{V^{*}(x, z)}(x)$ runs in time polynomial in $|x|$, and the ensembles $\left\{\operatorname{View}_{V^{*}}^{P}(x, z)\right\}_{x \in L, z \in\{0,1\}^{*}}$ and $\left\{S^{V^{*}(x, z)}(x)\right\}_{x \in L, z \in\{0,1\}^{*}}$ are computationally indistinguishable as a function of $|x|$.

Other Primitives. In our construction of zero-knowledge arguments we use a few other primitives including Witness-Indistinguishable (WI) Proofs [FS90, Proofs of Knowledge (POK) [FS90, BG02, and Special-Sound (SS) Proofs [CDS94. Due to lack of space, we refer the readers to the full version of this paper for a more detailed description of these primitives.

\section{Construction}

We define a zero-knowledge argument PARALlelZK in Section 3.1, and show that it satisfies Theorem 1 in Section 3.2 .

\subsection{The Protocol}

Our ZK argument PARALLELZK (also used in [PV08, PTV10]) is a slight variant of the precise ZK protocol of [MP06], which in turn is a generalization of the Feige-Shamir protocol [FS89]. The protocol for language $L \in N P$ proceeds in three stages, given a security parameter $n$, a common input statement $x \in$ $\{0,1\}^{n}$, and a round-parameter $m$ : 
Stage Init: The verifier picks two random strings $r_{1}, r_{2} \in\{0,1\}^{n}$ and sends their images $c_{1}=f\left(r_{1}\right), c_{2}=f\left(r_{2}\right)$ through a one-way function $f$ to the prover. The verifier then acts as the prover in $m$ parallel instances of a 4 round witness indistinguishable and special sound proof of knowledge (WI and SS-POK) of the NP statement " $c_{1}$ or $c_{2}$ is in the image set of $f$ " (a witness here would be a pre-image of $c_{1}$ or $c_{2}$ ). All but the last two messages of each SS-POK is exchanged in this stage; we denote their partial transcripts by $\left(\boldsymbol{\alpha}_{1}, \boldsymbol{\alpha}_{2}, \ldots, \boldsymbol{\alpha}_{m}\right)$.

Stage 1: $m$ rounds of message exchanges occur in Stage 1. In the $j^{\text {th }}$ round, the prover sends $\beta_{j}$, a random second last message of the $j^{\text {th }}$ SS-POK, and the verifier replies with the last message $\gamma_{j}$ of the proof. These $m$ rounds are called slots. Slot $i$ is convincing if the verifier produces an accepting proof (i.e., the transcript $\left(\boldsymbol{\alpha}_{i}, \beta_{i}, \gamma_{i}\right)$ is accepting). If there is ever an unconvincing slot, the prover aborts the whole session.

Stage 2: The prover provides a 4-round witness indistinguishable proof of knowledge (WI-POK) of knowledge of the statement " $x \in L$, or one of $c_{1}$ or $c_{2}$ is in the image set of $f^{\prime \prime}$.

Completeness and soundness follows directly from the proof of Feige and Shamir [FS89]; in fact, the protocol is an instantiation of theirs. Intuitively, to cheat in the protocol a prover must "know" an inverse to $c_{1}$ or $c_{2}$ (because Stage 2 is an argument of knowledge), which requires the prover to invert the one-way function $f$ (it is shown in FS90 that Stage Init and Stage 1 of the protocol cannot aid the prover in inverting $f$ ). A formal description of protocol ParallelZK is shown in Figure 1 .

Remark 4. We note that here we use multiple slots to improve the knowledge tightness of parallel zero knowledge, whereas previously, multiple slots was typically used to achieve concurrent zero knowledge and $\omega(\log n)$ slots were considered. In contrast, we show that in the context of parallel zero knowledge, using even constant number of slots improves the knowledge tightness significantly. Indeed, both our simulation technique and its analysis presented in the next section are new, where we rewind each slot to resolve all sessions in parallel (as opposed to previous works that focused on one session at a time).

\subsection{The Simulator}

To show that protocol $\Pi=$ PARALLELZK satisfies Theorem 1 given any polynomially bounded $k=k(n)$, we need to construct a black-box zero-knowledge simulator $S=S_{k}$ for protocol $\Pi^{k}$ (PARALLELZK repeated $k$ times in parallel). On a very high-level, our simulator follows that of Feige and Shamir [FS90]: after fixing the SS-POK prefixes in Stage Init, the simulator rewinds one of the "slots" in Stage 1 (the last two messages of the SS-POKs). If the verifier responds with two convincing slots, the simulator uses the special-soundness property to extract a "fake witness" $r$ such that $f(r)=c_{1}$ or $c_{2}$, and uses this fake witness to simulate Stage 2 of the protocol. 
Common Input: an instance $x$ of a language $L$ with witness relation $R_{L}$.

Auxiliary Input for Prover: a witness $w$, such that $(x, w) \in R_{L}(x)$.

Stage Init:

V uniformly chooses $r_{1}, r_{2} \in\{0,1\}^{n}$.

$\mathrm{V} \rightarrow \mathrm{P}: c_{1}=f\left(r_{1}\right), c_{2}=f\left(r_{2}\right)$.

$\mathrm{V} \leftrightarrow \mathrm{P}$ : Exchange in parallel (interactively) all but the last two messages $\boldsymbol{\alpha}_{1}, \ldots, \boldsymbol{\alpha}_{m}$ of $m \mathrm{WI}$ and SS-POKs on common input $\left(c_{1}, c_{2}\right)$ with respect to the witness relation:

$$
R_{L^{\prime}}\left(c_{1}, c_{2}\right)=\left\{r: f(r)=c_{1} \text { or } c_{2}\right\}
$$

Note that $\mathrm{V}$ acts as the prover in these SS-POK's.

Stage 1: For $j=1$ to $m$, exchange the $i^{\text {th }}$ "slot"

$\mathrm{P} \rightarrow \mathrm{V}$ : The second last message $\beta_{i}$ of the $i^{\text {th }}$ SS-POK.

$\mathrm{V} \rightarrow \mathrm{P}:$ The last message $\gamma_{i}$ of the $i^{\text {th }}$ SS-POK.

$\mathrm{P}$ aborts if $\left(\boldsymbol{\alpha}_{i}, \beta_{i}, \gamma_{i}\right)$ is not a valid SS-POK.

Stage 2:

$\mathrm{P} \leftrightarrow \mathrm{V}$ : a 4-round computational-WI proof of knowledge from $P$ to $V$ on common input $\left(c_{1}, c_{2}, x\right)$ with respect to the witness relation:

$$
R_{L^{\prime} \vee L}\left(c_{1}, c_{2}, x\right)=\left\{(r, w): r \in R_{L^{\prime}}\left(c_{1}, c_{2}\right) \text { or } w \in R_{L}(x)\right\}
$$

Fig. 1. PARAllelZK: a ZK argument for NP with round parameter $m$

Given an adversarial verifier $V^{*}$ (for protocol $\Pi^{k}$ ) and a common input $x \in$ $\{0,1\}^{n}$, the simulator $S^{V^{*}}(x)$ does the following:

1. The simulator $S$ interacts with $V^{*}$, following the honest prover strategy, until the end of Stage 1. We call this the reference simulation.

2 . The simulator $S$ attempts to resolve all $k$ parallel sessions in the reference simulation by extracting a fake witness $r$ from the SS-POKs for each nonaborting session; aborted sessions are automatically considered resolved (and no fake witnesses are needed). To do so, $S$ repeats the following step (called a rewinding pass) as many times as necessary, until all sessions are resolved.

3. A rewinding pass. For each slot $i$, the simulator rewinds the reference simulation back to the beginning of slot $i$, sends $V^{*}$ a fresh random message $\beta_{i}^{\prime}$, and receives a new reply $\gamma_{i}^{\prime}$ (of course this is done in parallel for all $k$ sessions). Note that for each unresolved session $j, S$ already knowns an accepting transcript $\left(\boldsymbol{\alpha}_{i}, \beta_{i}, \gamma_{i}\right)$ of SS-POK from the reference simulation. If session $j$ does not abort during slot $i$ in this rewinding pass, then $S$ learns another accepting transcript $\left(\boldsymbol{\alpha}_{i}, \beta_{i}^{\prime}, \gamma_{i}^{\prime}\right)$ of SS-POK. In this case, $S$ can resolve the session $j$ by extracting a fake witness using the special-sound property.

4. $S$ completes the reference simulation using extracted fake witnesses to simulate the Stage 2 proof (only needed in each parallel session that did not abort). $S$ outputs the view of $V^{*}$ on the reference simulation and this completion. 
For simplicity, we assume that for sessions that did not abort in the reference simulation, the extraction of fake witnesses always succeeds whenever $S$ receives an accepting slot in a rewinding pass (i.e., we assume that $S$ never sends the same value for $\beta$ twice). This assumption can be made without loss of generality by the following modifications of the simulation strategy.

- Let the simulator $S$ performs at most $2^{n}$ rewinding passes. If there exist any unsolved sessions $j$ after $2^{n}$ rewinding passes, $S$ resolves the session by brute force, i.e., by directly inverting the one-way function $f$ to obtain a fake witness of length $n$. This modification increases the running time (but not the number of queries) of $S$ by at most a poly $(n)$ factor (multiplicatively), and makes sure that $S$ makes at most poly $\left(2^{n}\right)$ queries to $V^{*}$.

- Let the final verifier challenge in the SS-POK have length $|\beta|=n^{2}$. In this case, the probability of $S$ ever querying $V^{*}$ with the same value of $\beta$ twice is poly $\left(2^{n}\right) \cdot 2^{-n^{2}}=2^{-\Omega\left(n^{2}\right)}$, definitely negligible in $n$.

We now show two lemmas regarding $S$ that together show that PARALLELZK is zero-knowledge when composed in parallel.

Lemma 5. $S$ runs in expected polynomial time, and makes $O\left(m k^{1 / m} \log ^{2} n\right)$ queries in expectation.

Lemma 6. On common input $x \in L$, the output of $S$ is indistinguishable from the real view of $V^{*}$.

We give a sketch of proof of Lemma 6first, and then prove Lemma 5 by bounding the expected number of rewinding passes before $S$ extracts all necessary fake witnesses.

Proof (Proof Sketch of Lemma 6). The output of $S$ up to the end of Stage 1 (i.e., the reference simulation) is identical to the view of $V^{*}$, because $S$ follows the honest prover strategy. The output of $S$ in Stage 2 of the protocol is computationally indistinguishable from the view of $V^{*}$ because the Stage 2 proof is witness indistinguishable. Formally, this can be shown with a hybrid argument where we incrementally exchange each of the $k$ parallel Stage 2 proofs from using "fake witnesses" $r$ such that $f(r)=c_{1}$ or $c_{2}$ (the simulator strategy), to a real witnesses $w$ for $x \in L$ (the honest prover strategy).

Proof of Lemma 5. We proceed to prove Lemma 5] by bounding the expected number of rewinding passes in an execution of $S$. Let $R$ be a random variable that denotes the number of rewinding passes. We will show that:

$$
\mathbb{E}[R]=\mathbb{E}[\# \text { rewinding passes }] \leq O\left(k^{1 / m} \cdot \log ^{2} n\right) .
$$

This then implies Lemma 5 because outside of rewinding passes, $S^{V *}(x)$ makes only $O(m)$ queries to $V^{*}$ and runs in polynomial time.

Before presenting our analysis for the general case of $m$ slots, we revisit the classical analysis for the case of single slot for intuition. 
The case of single slot. The analysis is very simple. For every $j \in[k]$, let $R_{j}$ denote the number of rewinding passes to resolve session $j$, and let $p_{j}$ be the probability that session $j$ does not abort during the single slot. Recall that session $j$ is resolved if it aborts in the reference simulation, and otherwise, the simulator needs to rewind the slot several times until session $j$ does not abort again. Hence, the expected number of rewinding passes to resolve session $j$ is

$$
\mathbb{E}\left[R_{j}\right]=\left(1-p_{j}\right) \cdot 0+p_{j} \cdot \frac{1}{p_{j}}=1
$$

By linearity of expectation, the expected number of rewinding passes is

$$
\mathbb{E}[R]=\sum_{j} \mathbb{E}\left[R_{j}\right]=k \leq O\left(k \cdot \log ^{2} n\right) .
$$

We note that the above simple analysis is tight. Consider the case where during the slot, each session aborts independently with probability $(1-1 / k)$. It is not hard to see that in this case, with constant probability, at least one session does not abort during the slot, and the simulator needs to rewind $k$ times in expectation to resolve the survival session. Therefore, the expected number of rewinding passes is $\Omega(k)$.

In fact, it is instructive to note that the following natural generalization of the above example is essentially the worse-case example for the general case of $m$ slots: during each slot $i \in[m]$, each survival session $j$ aborts independently with probability $\left(1-k^{-1 / m}\right)$. In this case, each session does not abort during the $m$ slots with probability $\left(k^{-1 / m}\right)^{m}=1 / k$, and hence with constant probability, at least one session survives after $m$ slots. Resolving the survival session requires $k^{1 / m} / m$ rewinding passes in expectation, and hence the expected number of rewinding passes is $\Omega\left(k^{1 / m} / m\right)$.

We note that although in the above example, each session aborts during each slot independently, in general, the aborting probability of each session at each slot can depends arbitrarily on the history and correlated arbitrarily.

The general case of $m$ slots. To analyze the expected number of rewinding passes, we define the following $[0,1]$-valued random variables based on the reference simulation generated in Step 1 . Let $h_{i}$ denote the partial transcript of the reference simulation before slot $i$. For every slot $i \in[m]$ and session $j \in[k]$, we define random variable $p_{i, j}$ as follows.

- If session $j$ is already aborted at the end of slot $i$, then we define $p_{i, j} \triangleq 1$.

- Otherwise, we define $p_{i, j}$ to be the conditional probability

$$
p_{i, j} \triangleq \operatorname{Pr}\left[\operatorname{session} j \text { does not abort during slot } i \mid h_{i}\right] \text {. }
$$

For intuition, $p_{i, j}$ is essentially the probability that $S$ can resolve session $j$ by rewinding slot $i$. Now consider the best slot for each session - the slot with the highest $p_{i, j}$ value (this is the slot that $S$ wants to rewind). We record this value as

$$
p_{j}^{*}=\max _{i} p_{i, j}
$$


Note that for a session $j$ that aborts in the reference simulation, we have $p_{j}^{*}=1$, indicating that sessions $j$ is already resolved and matching the above intuition. Finally, the number of rewinding passes depends heavily on the worst session the session with the worst $p_{j}^{*}$ value (the "worst best slot"). We record this value as the critical probability:

$$
p^{*}=\min _{j} p_{j}^{*} .
$$

To see how the critical probability $p^{*}$ plays an important role in the expected number of rewinding passes, note that on one hand, $S$ needs roughly $1 / p^{*}$ rewinding passes to resolve the worse-case session; on the other hand, the chance of having a reference simulation with small critical probability (say, $p^{*} \leq p$ ) is rare (at most $p^{m}$ ). Therefore, to upper bound $\mathbb{E}[R]$, we define the following events, which partition the probability space according to the critical probability. For every $t \in \mathbb{N}$, let

$$
\alpha_{t} \stackrel{\text { def }}{=}\left(\frac{1}{2^{t} \cdot k^{1 / m}}\right)
$$

- Let $A_{0}$ be the event that $p^{*} \geq \alpha_{0}=k^{-1 / m}$, and for every $t \in \mathbb{N}$, let $A_{t}$ be the event that

$$
\alpha_{t} \leq p_{j}^{*}<\alpha_{t-1} .
$$

Similarly for every session $j \in[k]$,

- Let $A_{0, j}$ be the event that $p_{j}^{*} \geq \alpha_{0}=k^{-1 / m}$, and for every $t \in \mathbb{N}$, let $A_{t, j}$ be the event that

$$
\alpha_{t} \leq p_{j}^{*}<\alpha_{t-1}
$$

We can now express the expectation of the number of rewinding passes as follows.

$$
\begin{aligned}
\mathbb{E}[R] & =\sum_{t \geq 0} \operatorname{Pr}\left[A_{t}\right] \cdot \mathbb{E}\left[R \mid A_{t}\right] \\
& \leq \operatorname{Pr}\left[A_{0}\right] \cdot \mathbb{E}\left[R \mid A_{0}\right]+\sum_{t \geq 1}\left(\sum_{j=1}^{k} \operatorname{Pr}\left[A_{t, j}\right]\right) \cdot \mathbb{E}\left[R \mid A_{t}\right],
\end{aligned}
$$

where the last inequality follows by $A_{t} \subseteq \cup_{j} A_{t, j}$ (which follows from definition). We proceed to bound each term. For $A_{0}$, we use trivial bound $\operatorname{Pr}\left[A_{0}\right] \leq 1$. For general $t \geq 1$ and every $j \in[k]$, we first observe that when $A_{t, j}$ happens, session $j$ does not abort all of its $m$ slots in the reference simulation (since otherwise, $\left.p_{j}^{*}=1\right)$. This happened despite the fact that each slot $i$ in session $j$ in the reference simulation could have only survived (not aborted) with probability $p_{i, j} \leq \alpha_{t-1}$. Thus,

$$
\operatorname{Pr}\left[A_{t, j}\right] \leq \alpha_{t-1}^{m}=\left(\frac{1}{2^{t-1} \cdot k^{1 / m}}\right)^{m}=\frac{1}{2^{m(t-1)} \cdot k},
$$

and,

$$
\sum_{j=1}^{k} \operatorname{Pr}\left[A_{t, j}\right] \leq k \cdot \frac{1}{2^{m(t-1)} \cdot k}=\frac{1}{2^{m(t-1)}}
$$


It remains to bound $\mathbb{E}\left[R \mid A_{t}\right]$, which is given in the follow lemma.

Lemma 7. For every $t \geq 0$, we have

$$
\mathbb{E}\left[R \mid A_{t}\right] \leq O\left(2^{t} \cdot k^{1 / m} \cdot \log ^{2} n\right) .
$$

We apply Lemma $[$ to upper bound $\mathbb{E}[R]$ first.

$$
\begin{aligned}
\mathbb{E}[R] & \leq \mathbb{E}\left[R \mid A_{0}\right]+\sum_{t \geq 1} \frac{1}{2^{m(t-1)}} \cdot \mathbb{E}\left[R \mid A_{t}\right] \\
& \leq O\left(k^{1 / m} \cdot \log ^{2} n\right)+\sum_{t \geq 1} \frac{2^{t}}{2^{m(t-1)}} \cdot O\left(k^{1 / m} \cdot \log ^{2} n\right) \\
& \leq O\left(k^{1 / m} \cdot \log ^{2} n\right) .
\end{aligned}
$$

This completes the proof of Lemma 5.

Proof (Proof of Lemma 7). The event $A_{t}$ means that in the reference simulation, for every non-aborting session $j$, there exists a useful slot $i \in[m]$ such that

$$
\operatorname{Pr}\left[\operatorname{session} j \text { is not aborted after slot } i \mid h_{i}\right]=p_{i, j} \geq \alpha_{t} \text {. }
$$

Therefore, in each rewinding pass, the simulator $S$ may learn an (additional) accepting transcript of SS-POK in session $j$ with probability at least $\alpha_{t}$, allowing it to extract a fake witness.

Fix a non-aborting session $j$, and define

$$
q=\left(\frac{10 \cdot \log ^{2} n}{\alpha_{t}}\right)=O\left(2^{t} \cdot k^{1 / m} \cdot \log ^{2} n\right),
$$

Because the rewinding passes are independent, we have

$\operatorname{Pr}\left[\operatorname{session} j\right.$ is resolved after $q$ rewinding passes] $=1-\left(1-\alpha_{t}\right)^{q} \geq 1-\mathrm{ngl}(\mathrm{n})$.

Since there are at most $k$ survival sessions, by the union bound,

$\operatorname{Pr}[$ all sessions are resolved after $q$ rewinding passes $] \geq 1-\operatorname{ngl}(\mathbf{n})$.

In other words, every $q$ rewinding passes can solve all the sessions with probability at least $1-\mathrm{ngl}(\mathrm{n})$. It follows that

$$
\begin{aligned}
\mathbb{E}\left[R \mid A_{t}\right] & \leq(1-\operatorname{ngl}(\mathrm{n})) \cdot q+\operatorname{ngl}(\mathrm{n})(1-\mathrm{ngl}(\mathrm{n})) \cdot 2 q+\cdots \\
& \leq O(q)=O\left(2^{t} \cdot k^{1 / m} \cdot \log ^{2} n\right)
\end{aligned}
$$




\section{Lower Bound}

The proof of Theorem 2 follows a well-known framework (e.g., GK96b, CKPR01). Let $S$ be a black-box zero-knowledge simulator for $\Pi^{k}=\left(P^{k}, V^{k}\right)$ that makes less than $q=O\left(k^{1 / m} / \log ^{2} n\right)$ queries, and let $V^{k *}$ be a particular adversarial verifier to be specified later. We define $\mathcal{D}$, a BPP decision procedure for $L$ by combining $S$ and $V^{k *}$ : on input instance $x, \mathcal{D}(x)$ accepts if and only if $S^{V^{k *}}(x)$ outputs an accepting view of $V^{k *}$ (i.e., all $k$ sessions of $V^{k *}$ accept). Using the zero-knowledge property, it is easy to show (see for example GK96b]) that if the modified protocol $\Pi^{k *}=\left(P^{k}, V^{k *}\right)$ is complete for $L$ (based on our choice of $V^{k *}$ ), then $\mathcal{D}$ is complete for $L$ as well. The main effort of the proof is to show that $\mathcal{D}$ is sound; this relies both on the choice of $V^{k *}$ and the fact that $S$ makes less than $q$ queries to $V^{k *}$. We discuss our choice of $V^{k *}$ in Section 4.1 , and analyze the soundness of $\mathcal{D}$ in Section 4.2

\subsection{The Random Termination Verifier $V^{k *}$}

In this section, we define a verifier $V^{k *}$ for the parallelized protocol with two goals in mind: the protocol $\Pi^{k *}=\left(P^{k}, V^{k *}\right)$ should be complete (so that $\mathcal{D}$ is complete), and $V^{k *}$ should be sound against any rewinding simulator $S$ that makes less than $q$ queries to $V^{k *}$ (so that $\mathcal{D}$ is sound).

Just as CKPR01, we define $V^{k *}$ to follow the honest verifier strategy $V^{k}$ with one extra property: random termination 2 Whenever the prover $P^{k}$ or the rewinding simulator $S$ makes a query to $V^{k *}, V^{k *}$ determines, with independent and fresh randomness 3 whether or not to terminate immediately and accept with probability $\rho \in[0,1]$, a parameter to be specified later; this is done independently for each of the $k$ parallel sessions (i.e., one session may be terminated while other sessions continue). Due to this independence among parallel sessions, we often treat $V^{k *}$ as $k$ machines, $\left(V_{1}^{*}, \ldots, V_{k}^{*}\right)$, each responsible for making the decision to terminate and generating the verifier messages for one session. Note that the fresh randomness is only used to decide whether to terminate or not; $V^{k *}$ generates protocol messages using its default random tape that is kept the same between rewinds (as expected by following the honest verifier strategy).

Clearly, $\Pi^{k *}=\left(P^{k}, V^{k *}\right)$ is still complete. It remains to show that $V^{k *}$ is "sound" against the rewinding $S$; that is, on input $x \notin L, S^{V^{k *}}$ is unlikely to

${ }^{2}$ The term "random termination" was first used by Haitner Hai09, but the random termination verifier we considered already appeared in the earlier work of [CKPR01].

${ }^{3}$ We use a well-known technique (see for example GK96b, CKPR01]) to generate fresh independent randomness on the fly for each query from the simulator $S$, despite the fact that $S$ may rewind $V^{k *}$ between queries and force $V^{k *}$ to use the same random tape. Let $\mathcal{H}$ be a family of $q$-wise independent hash-functions, and let $V^{k *}$ sample one hash-function $h \leftarrow \mathcal{H}$ in the very beginning. Then whenever $V^{k *}$ receives a query (from $P^{k}$ or $S$ ), $V^{k *}$ applies $h$ to the current protocol transcript (the sequence of messages exchanged in the protocol so far) and use the output as a fresh random tape. Since $S$ makes at most $q$ queries to $V^{k *}$, the output distribution of the hashfunction is truly uniformly random. 
generate an accepting transcript of $V^{k *}$. From now on we drop the common input $x \notin L$. Intuitively, by randomly terminating, $V^{k *}$ can better protect its

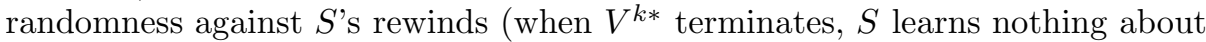
$V^{k *}$ 's fixed random tape), thus ensuring soundness. To make this intuition more concrete, suppose for example that $S$ made $q$ queries $\tau_{1}, \ldots, \tau_{q}$ to $V^{k *}$, and without loss of generality outputs the view of $V^{k *}$ on a subset of size $m$ of those queries $4, T=\left\{\tau_{i_{1}}, \ldots, \tau_{i_{m}}\right\}$. Further suppose that there exists a parallel session $j \in[k]$ such that $V^{k *}$ does not terminate on the queries in $T$, but terminates on all remaining queries. Then intuitively, $S$ 's rewinding does not help $S$ convince $V^{k *}$ in session $j$, and the soundness of the original protocol $\Pi$ should imply that $V^{k *}$ rejects with overwhelming probability in session $j$ (and therefore rejects overall).

The core of our proof is to show that, with high probability, for every subset of size $m$ of queries $T=\left\{\tau_{i_{1}}, \ldots, \tau_{i_{m}}\right\}$ made by $S$, there exists a session $j \in[k]$ with overwhelming probability such that rewinds are "not helpful" for session $j$ with respect to $T$ in the above manner. We make this possible by setting the termination probability to $\rho=(1-1 / q)$.

We now state the formal lemmas. Let $n$ be the security parameter and $L$ be a language. Suppose there exists a $m(n) \in O\left(\frac{\log n}{\log \log n}\right)$-round $\operatorname{argument} \Pi=$ $(P, V)$ for $L$ with perfect completeness and negligible soundness error. For any polynomially bounded $k(n) \geq n$, let $S$ be a black-box zero-knowledge simulator of the parallelized protocol $\Pi^{k}=\left(P^{k}, V^{k}\right)$ that makes at most

$$
q=k^{1 / m} /\left(\log ^{2} n\right)
$$

queries, and let $V^{k *}$ be a random termination verifier of the parallelized protocol with termination probability

$$
\rho=\left(1-\frac{1}{q}\right)=\left(1-\frac{1}{k^{1 / m}} \cdot\left(\log ^{2} n\right)\right) .
$$

(These parameters passes the following sanity checks: $q$ is polynomially bounded and $q \geq m$ - the simulator queries $V^{k *}$ at least once for each round of the protocol. It is also useful later to know that $\left(\begin{array}{c}q \\ m\end{array}\right) \leq q^{m} \leq k$.) Then:

Lemma 8. On input $x \in L, \mathcal{D}(x)$ accepts with probability 1, i.e., $S^{V^{* *}}(x)$ outputs an accepting view of $V^{k *}$ with probability $1-\mathrm{ngl}(\mathrm{n})$.

Lemma 9. On input $x \notin L$, the probability that $S^{V^{k *}}(x)$ generates an accepting view of $V^{k *}$ is negligible, i.e., $\mathcal{D}$ has negligible soundness error.

We sketch the proof of Lemma 8 now, and give the proof of Lemma 9 in the next section.

\footnotetext{
${ }^{4}$ Without loss of generality, we may assume that before $S$ outputs a view of $V^{k *}$, $S$ first queries $V^{k *}$ with the messages in the view (if $S$ hasn't already). This may increase the number of queries by $m$, and thus weaken the resulting lower bound from $q$ to $q-m$. Nevertheless, this does not change our lower bound since $q=\omega(m)$ in Theorem 2 .
} 
Proof (Proof Sketch). Using the zero-knowledge property, the output of $S$ is indistinguishable from the view of $V^{k *}$ in an execution with $P^{k}$. Therefore it is enough to show that $\left\langle P^{k}, V^{k *}\right\rangle(x)$ accepts with probability 1 . In each parallel session $j \in[k], V_{j}^{*}$ accepts by definition if it decides to terminate in some protocol round. Otherwise, $V_{j^{*}}$ is identical to $V$ and would still accept with probability 1 because the original protocol $\Pi=(P, V)$ has perfect completeness.

\subsection{Soundness of $\mathcal{D}$}

Proof (Proof of Lemma 9). We prove Lemma9 with a reduction. Suppose for the sake of contradiction that $S$ convinces $V^{k *}$ on some input $x \notin L$ with probability more than $1 / p(n)$ for some polynomial $p$. Using $S$, we construct a cheating prover $P^{*}$ for the original protocol $\Pi=(P, V)$ that convinces $V$ with non-negligible probability.

Before we start, assume without loss of generality that $S$ makes exactly $q$ queries, and that before $S$ outputs a view of $V^{k *}, S$ would first query $V^{k *}$ on all previous messages in the view. For technical convenience, we let $V^{k *}$ make a fresh decision to terminate for each query and each session, even if $V^{k *}$ has already terminated previously in the same session. I.e., regardless of history or message content, for each query and each parallel session, $V^{k *}$ always terminates independently with probability $\rho$.

Our $P^{*}$ is a natural extension of the classic reduction of GK96b $-P^{*}$ guesses a session $j_{0} \in[k]$ and $m$ indices $T_{0}=\left\{i_{1}, \ldots, i_{m}\right\} \subseteq[q]$ uniformly at random, and interacts with an outside honest $V$ by internally simulating an interaction of $\left(S, V^{k *}\right)$ with $V$ embedded in session $j_{0}$, queries $\tau_{i_{1}}, \ldots, \tau_{i_{m}}$ of $V^{k *}$. In comparison, the idea of guessing a random query subset is exactly as in GK96b. The difference is that the reduction in GK96b is for single session protocols, and in contrast, we reduce from parallel protocols to single session protocols. Hence, our reduction $P^{*}$ guesses a random session as well.

In more details, $P^{*}$ runs $S$ and $V^{k *}$ internally. It simulates $k-1$ sessions of $V^{k *}$ honestly (except $V_{j_{0}}^{*}$ ). When simulating $V_{j_{0}}^{*}$ for the $i^{\text {th }}$ query where $S$ queries $\tau_{i}, P^{*}$ first simulates (with fresh randomness) $V_{j_{0}}^{*}$ 's decision on termination. If $V_{j_{0}}^{*}$ decides to terminate but $i \in T_{0}$ or if $V_{j_{0}}^{*}$ does not terminate but $i \notin T_{0}, P^{*}$ aborts (in both these cases, the termination decision of $V_{j_{0}}^{*}$ is incompatible with $P^{*}$ 's choice of queries to forward). If the forwarded queries (index set $T_{0}$ ) are not "consistent" (e.g., if they query for the same round of the protocol more than once, or the query contains inconsistent transcript), $P^{*}$ aborts as well. Note that if $P^{*}$ does not abort, then $V^{k *}$ is perfectly simulated (even in session $j_{0}$ ).

Now consider the following best case scenario. Suppose that at the end of the simulation, $S$ successfully outputs an accepting view of $V^{k *}$. Moreover, suppose that the accepting view consists exactly of the queries in index set $T_{0}$ (this automatically guarantees that the forwarded queries are consistent), and suppose that $P^{*}$ does not abort (i.e., termination decisions are compatible with the forwarded queries). Then, $P^{*}$ will have successfully convinced the outside honest $V$. The rest of the proof is devoted to show that this best case scenario occurs with noticeable probability (roughly $1 /\left(p \cdot k^{2}\right)$ ). 
Let $T \subset[q]$ denote an index set $\left\{i_{1}, \ldots, i_{m}\right\}$ of size $m$. For an index set $T \subset[q]$ and a session $j \in[k]$, we define $A(T, j)$ to be the event that, on session $j, V^{k *}$ terminates session $j$ on query $\tau_{i}$ iff $i \notin T$. Referring back to our intuition earlier, $A(T, j)$ denotes the event that for session $j, S$ 's rewinds are not helpful with respect to the queries indexed by $T$. If event $A(T, j)$ holds, and $S$ uses the queries indexed by $T$ to form an accepting view of $V^{k *}$, and $P^{*}$ guesses both $T_{0}=T$ and $j_{0}=j$ in the beginning, then $P^{*}$ will have successfully convinced the outside honest $V$.

We claim that by the setting of parameters, we have

$$
\operatorname{Pr}[\forall T \subset[q], \exists j \in[k] \text { s.t. } A(T, j)] \geq 1-\operatorname{ngl}(\mathbf{n})
$$

where $\mathrm{ngl}(\mathrm{n})$ denotes a negligible quantity in $n$. In words, with overwhelming probability, for every possible index set $T$ of size $m$ that $S$ may use to output a view of $V^{k *}$, there exists a session $j$ such that $S$ 's rewinds are not helpful with respect to the queries indexed by $T$.

Before proving (11), we first use the claim to show that $P^{*}$ convinces $V$ with noticeable probability. Recall that $S$ outputs an accepting view of $V^{k *}$ with probability $1 / p$. By a union bound, we have

$$
\begin{aligned}
& \operatorname{Pr}\left[\left(S \text { outputs accepting view of } V^{k *}\right) \wedge(\forall T \subset[q], \exists j \in[k] \text { s.t. } A(T, j))\right] \\
& \geq(1 / p)-\operatorname{ngl}(\mathrm{n}) .
\end{aligned}
$$

Note that when the above event holds, there exist a unique index $\hat{T}$ of $m$ queries used by $S$ to form an accepting view of $V^{k *}$, and there exists a session $\hat{j} \in[k]$ such that $A(\hat{T}, \hat{j})$ holds. As mentioned earlier, if $P^{*}$ guesses $j_{0}=\hat{j}$ and $T_{0}=$ $\hat{T}$ correctly, $P^{*}$ will have successfully convinced $V$. Since $P^{*}$ guesses $j$ and $T$ uniformly at random and independent of the interaction between $S$ and $V^{k *}$, we have

$$
\begin{aligned}
& \operatorname{Pr}\left[P^{*} \text { convinces } V\right] \\
& \geq \operatorname{Pr}\left[\left(S \text { convinces } V^{k *}\right) \wedge(\forall T \subset[q], \exists j \in[k] \text { s.t. } A(T, j))\right. \\
& \left.\quad \wedge\left(P^{*} \text { guesses } \hat{T} \text { and } \hat{j} \text { correctly }\right)\right] \\
& \geq \frac{(1 / p-\operatorname{ngl}(\mathrm{n}))}{k \cdot\left(\begin{array}{c}
q \\
m
\end{array}\right)} \geq \frac{1}{p \cdot k^{2}},
\end{aligned}
$$

where in the last line we used $\left(\begin{array}{c}q \\ m\end{array}\right) \leq q^{m} \leq k$. This contradicts to the fact that $\Pi$ has negligible soundness error and completes our analysis.

It remains to show (11). By definition, each session $j$ terminates on each query $\tau_{i}$ with probability exactly $\rho$, independent from any other session or query. Hence, for any session $j$ and index set $T$ of size $m$, the probability that event $A(T, j)$ holds is

$$
\operatorname{Pr}[A(T, j)]=\rho^{q-m} \cdot(1-\rho)^{m} \geq\left(1-\frac{1}{q}\right)^{q} \cdot\left(\frac{1}{q}\right)^{m} \geq \Omega\left(\frac{1}{k} \cdot\left(\log ^{2 m} n\right)\right) .
$$


It follows that

$$
\operatorname{Pr}[\exists j \in[k] \text { s.t. } A(T, j)] \geq 1-\left(1-\Omega\left(\frac{1}{k} \cdot\left(\log ^{2 m} n\right)\right)\right)^{k} \geq 1-e^{-\Omega\left(\log ^{2 m} n\right)} .
$$

Finally, by a union bound, we have

$$
\operatorname{Pr}[\forall T \subset[q], \exists j \in[k] \text { s.t. } A(T, j)] \geq 1-e^{-\Omega\left(\log ^{2 m} n\right)} \cdot\left(\begin{array}{c}
q \\
m
\end{array}\right) \geq 1-\operatorname{ngl}(\mathbf{n}),
$$

as claimed.

As with most lower bounds for black-box zero-knowledge, a careful reading reveals that Theorem 2 also applies to more liberal definitions of zero-knowledge, such as $\varepsilon$-zero-knowledge and zero-knowledge with expected polynomial time simulators. Additionally, note that the proof of Lemma 9 never assume that $S$ is a zero-knowledge simulator, and works just as well for any PPT oracle machine $S$.

Remark 10. By examining the technical inner workings of the proof of Canetti, Kilian, Petrank and Rosen [CKPR01] (which also uses a random termination verifier), we discovered that part of their analysis implicitly presents a lower bound for the number of queries made by black-box simulators for parallel zeroknowledge protocols. Compared with Theorem 2 and our analysis, the result of CKPR01 establishes a weaker bound (and is arguably more complicated); this is not surprising, since establishing a parallel lower bound was not their goal.

Specifically, [CKPR01] implicitly establishes a $\log ^{\omega(1)}(k)$ lower bound on the number of simulator queries, whereas we were able to establish a lower bound of $k^{1 / m} /\left(\log ^{2} n\right)$. Nevertheless, we believe that by adapting our parameters (which may seem strange for their setting), their analysis could be strengthened to match our lower bounds (we have not verified all the details, however).

Acknowledgments. We thank to Iftach Haitner and Johan Håstad for useful discussion in the early stage of this research.

\section{References}

[Bar01] Barak, B.: How to go beyond the black-box simulation barrier. In: FOCS 2001, pp. 106-115 (2001)

[BG02] Barak, B., Goldreich, O.: Universal arguments and their applications. In: Computational Complexity, pp. 162-171 (2002)

[BL02] Barak, B., Lindell, Y.: Strict polynomial-time in simulation and extraction. In: STOC 2002, pp. 484-493 (2002)

[Blu86] Blum, M.: How to prove a theorem so no one else can claim it. In: Proc. of the International Congress of Mathematicians, pp. 1444-1451 (1986)

[CDS94] Cramer, R., Damgård, I., Schoenmakers, B.: Proof of Partial Knowledge and Simplified Design of Witness Hiding Protocols. In: Desmedt, Y.G. (ed.) CRYPTO 1994. LNCS, vol. 839, pp. 174-187. Springer, Heidelberg (1994) 
[CKPR01] Canetti, R., Kilian, J., Petrank, E., Rosen, A.: Black-box concurrent zeroknowledge requires $\tilde{\omega}(\log n)$ rounds. In: STOC 2001, pp. 570-579 (2001)

[DNS04] Dwork, C., Naor, M., Sahai, A.: Concurrent zero-knowledge. J. ACM 51(6), 851-898 (2004)

[FS89] Feige, U., Shamir, A.: Zero Knowledge Proofs of Knowledge in Two Rounds. In: Brassard, G. (ed.) CRYPTO 1989. LNCS, vol. 435, pp. 526-544. Springer, Heidelberg (1990)

[FS90] Feige, U., Shamir, A.: Witness indistinguishable and witness hiding protocols. In: STOC, pp. 416-426 (1990)

[GK96a] Goldreich, O., Kahan, A.: How to construct constant-round zero-knowledge proof systems for NP. Journal of Cryptology 9(3), 167-190 (1996)

[GK96b] Goldreich, O., Krawczyk, H.: On the composition of zero-knowledge proof systems. SIAM Journal on Computing 25(1), 169-192 (1996)

[GMR89] Goldwasser, S., Micali, S., Rackoff, C.: The knowledge complexity of interactive proof systems. SIAM Journal on Computing 18(1), 186-208 (1989)

[GMW91] Goldreich, O., Micali, S., Wigderson, A.: Proofs that yield nothing but their validity for all languages in NP have zero-knowledge proof systems. J. ACM 38(3), 691-729 (1991)

[GO94] Goldreich, O., Oren, Y.: Definitions and properties of zero-knowledge proof systems. Journal of Cryptology 7, 1-32 (1994)

[Gol01] Goldreich, O.: Foundations of Cryptography — Basic Tools. Cambridge University Press (2001)

[Gol02] Goldreich, O.: Concurrent zero-knowledge with timing, revisited. In: STOC 2002, pp. 332-340 (2002)

[Hai09] Haitner, I.: A parallel repetition theorem for any interactive argument. In: FOCS 2009, pp. 241-250 (2009)

[HRS09] Haitner, I., Rosen, A., Shaltiel, R.: On the (Im)Possibility of ArthurMerlin Witness Hiding Protocols. In: Reingold, O. (ed.) TCC 2009. LNCS, vol. 5444, pp. 220-237. Springer, Heidelberg (2009)

[Kat08] Katz, J.: Which Languages Have 4-Round Zero-Knowledge Proofs? In: Canetti, R. (ed.) TCC 2008. LNCS, vol. 4948, pp. 73-88. Springer, Heidelberg (2008)

[KPR98] Kilian, J., Petrank, E., Rackoff, C.: Lower bounds for zero knowledge on the internet. In: FOCS 1998, pp. 484-492 (1998)

[MP06] Micali, S., Pass, R.: Local zero knowledge. In: STOC 2006, pp. 306-315 (2006)

[PPS ${ }^{+}$08] Pandey, O., Pass, R., Sahai, A., Tseng, W.-L.D., Venkitasubramaniam, M.: Precise Concurrent Zero Knowledge. In: Smart, N.P. (ed.) EUROCRYPT 2008. LNCS, vol. 4965, pp. 397-414. Springer, Heidelberg (2008)

[PTV10] Pass, R., Tseng, W.-L.D., Venkitasubramaniam, M.: Eye for an Eye: Efficient Concurrent Zero-Knowledge in the Timing Model. In: Micciancio, D. (ed.) TCC 2010. LNCS, vol. 5978, pp. 518-534. Springer, Heidelberg (2010)

[PV08] Pass, R., Venkitasubramaniam, M.: On Constant-Round Concurrent ZeroKnowledge. In: Canetti, R. (ed.) TCC 2008. LNCS, vol. 4948, pp. 553-570. Springer, Heidelberg (2008)

[Ros00] Rosen, A.: A Note on the Round-Complexity of Concurrent Zero-Knowledge. In: Bellare, M. (ed.) CRYPTO 2000. LNCS, vol. 1880, pp. 451-468. Springer, Heidelberg (2000) 